\title{
Slow-light enhanced correlated photon pair generation in a silicon photonic crystal waveguide
}

\author{
C. Xiong, ${ }^{1, *}$ Christelle Monat, ${ }^{1,2}$ Alex S. Clark, ${ }^{1,3}$ Christian Grillet, ${ }^{1}$ Graham D. Marshall, ${ }^{4}$ M. J. Steel, ${ }^{4}$ Juntao Li, ${ }^{5}$ \\ Liam O'Faolain, ${ }^{5}$ Thomas F. Krauss, ${ }^{5}$ John G. Rarity, ${ }^{3}$ and Benjamin J. Eggleton ${ }^{1}$ \\ ${ }^{1}$ Centre for Ultrahigh-bandwidth Devices for Optical Systems (CUDOS), Institute of Photonics and Optical Science (IPOS), \\ School of Physics, University of Sydney, Sydney, NSW 2006, Australia \\ ${ }^{2}$ Institut des Nanotechnologies de Lyon, Ecole Centrale de Lyon, 36 Avenue Guy de Collongue, 69134 Ecully, France \\ ${ }^{3}$ Centre for Quantum Photonics, University of Bristol, Queens Building, University Walk, Bristol, BS8 1TR, UK \\ ${ }^{4}$ CUDOS, MQ Photonics Research Centre, Department of Physics \& Astronomy, Macquarie University, Sydney, NSW 2109, Australia \\ ${ }^{5}$ School of Physics and Astronomy, University of St. Andrews, Fife, KY16 9SS, UK \\ *Corresponding author: chunle@physics.usyd.edu.au
}

Received June 17, 2011; revised August 5, 2011; accepted August 5, 2011;

posted August 9, 2011 (Doc. ID 149441); published August 26, 2011

\begin{abstract}
We report the generation of correlated photon pairs in the telecom $C$-band at room temperature from a dispersionengineered silicon photonic crystal waveguide. The spontaneous four-wave mixing process producing the photon pairs is enhanced by slow-light propagation enabling an active device length of less than $100 \mu \mathrm{m}$. With a coincidence to accidental ratio of 12.8 at a pair generation rate of 0.006 per pulse, this ultracompact photon pair source paves the way toward scalable quantum information processing realized on-chip. (c) 2011 Optical Society of America

OCIS codes: $130.5296,270.5585,190.4380,190.4390$.
\end{abstract}

The integration of optical components for use in emerging quantum technologies such as quantum-secured communication [1] and quantum computation [2] is under intense investigation. A bright and low-noise singlephoton source in the low-loss telecom bands is a key requirement for many applications. In this context, single quantum emitters have been exploited to realize solidstate single-photon sources [3,4], but despite some recent advances, these approaches still present their own challenges, such as the need for low temperature, or the difficulty in integrating single emitters in practical devices. So far, the most common strategy to generate single photons relies on producing correlated photon pairs using a bulk optic nonlinear crystal. The photon pair generated by the underlying spontaneous nonlinear process allows for the detection of one photon heralding the arrival of the other. As nonlinear pair generation is stochastic, the photon states produced are nondeterministic and are often contaminated by states of higher photon number, reducing the fidelity of any quantum logic circuit using such states as inputs. Attenuating the power of the pump laser driving the nonlinear process reduces the fraction of higher number states, but at the expense of the source brightness. The development of a deterministic nonlinear single-photon source therefore requires many heralded sources that are pumped with sufficiently low power to preclude higher photon number states and that are multiplexed from a parallel architecture to guarantee the generation of single photons on command [톻]. To meet the demands of quantum information processing and its requirements of many single-photon input states and even cluster states [ㄱ,,$\underline{8}$, a mature source may eventually contain hundreds or even thousands of individual pair generation units combined with intelligent routing to collect the photons into a single mode for delivery to any photonic application. Achieving these functionalities in a scalable scheme requires the miniaturization of each individual unit so that they can be integrated on a photonic chip.
Schemes investigated for such sources include spontaneous three-wave mixing using periodically poled lithium niobate (PPLN) waveguides [9] and spontaneous fourwave mixing (SFWM) using chalcogenide glass rib waveguides [10] or silicon nanowires [11-14]. In each case, the flux of generated single photons grows with the effective interaction strength $\gamma P L$, where $P$ is the pump power, $L$ the device length, and $\gamma$ measures the strength of the optical nonlinearity. The required path lengths of PPLN and chalcogenide waveguides are typically a few centimeters. The use of highly nonlinear silicon nanowires decreases the device length to $\sim 1 \mathrm{~cm}$, but further reducing the device size has remained a challenge because of the requirement for increased device nonlinearity.

Recently it has been reported that photonic crystal (PhC) structures can dramatically enhance the effective nonlinearity of silicon waveguides through slowlight propagation [15-18]. In particular, the advent of dispersion-engineered slow-light waveguides has enabled the demonstration of broadband stimulated fourwave mixing in devices of $\sim 100 \mu \mathrm{m}$ length $[17,18], 2$ orders of magnitude shorter than silicon nanowires. In this Letter, we report the generation of $\sim 1550 \mathrm{~nm}$ correlated photon pairs in a low-loss silicon PhC waveguide that has been dispersion-engineered to exploit slow-light enhanced SFWM. This enhancement allows the length of the device to be reduced to $96 \mu \mathrm{m}$ while retaining sufficient SFWM efficiency, making this the most compact emitter of correlated photon pairs yet reported, to our knowledge.

The generation of correlated photon pairs through SFWM is illustrated in Fig. 1(a). A coherent pulse of light enters the $\mathrm{PhC}$ region, where two photons from the pump are converted to signal and idler photons of higher and lower frequencies, respectively, to form a quantum correlated state. Because of the slow-light effect of the $\mathrm{PhC}$, the pump travels at a group velocity of $\sim c / 30$, and is strongly spatially compressed. Compared with silicon nanowires, this enhances the nonlinear interaction per 

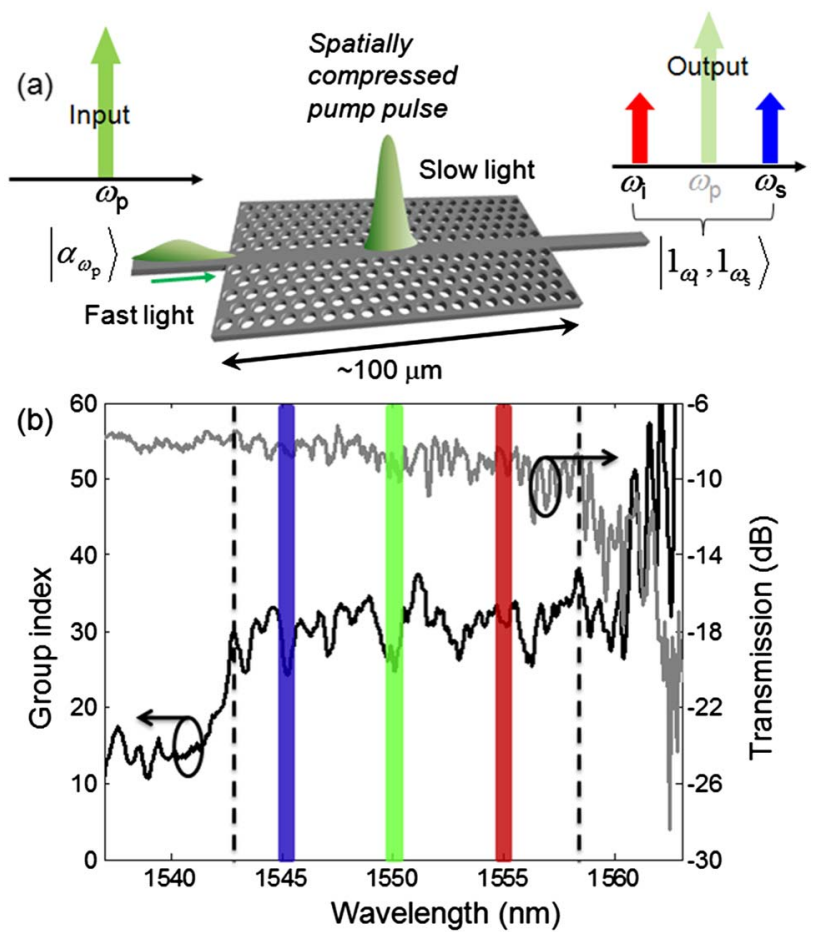

Fig. 1. (Color online) (a) Schematic of SFWM in a silicon slow-light PhC waveguide. (b) Group index and total transmission of light in the silicon $\mathrm{PhC}$ waveguide. The window (between dotted lines) with a flat group index of $\sim 30$ and slightly increased loss defines the slow-light regime. The pump, signal, and idler bands are represented by green (middle), blue (left), and red (right) lines, respectively.

unit length per unit pump power, $\gamma$, approximately tenfold, and in turn the SFWM efficiency, which is ideally proportional to $(\gamma P L)^{2}$, by 2 orders of magnitude [17].

The silicon slow-light device used in this study was fabricated on a silicon-on-insulator wafer comprising a $220 \mathrm{~nm}$ silicon layer on $2 \mu \mathrm{m}$ of silica through the use of electron beam lithography and reactive ion etching $[17,18]$. The PhC waveguide was created from a triangular lattice of air holes etched in a suspended silicon membrane with a row of holes missing along the $\Gamma K$ direction. The rows adjacent to the waveguide center were laterally shifted to engineer the waveguide dispersion such that it exhibits a group index of $\sim 30$ with low dispersion and moderate loss across a $15 \mathrm{~nm}$ window centered at $1553 \mathrm{~nm}$ [see Fig. 1(b)] [19]. Silicon access waveguides, including inverse tapers terminated by wide polymer waveguides, were added to the input and output of the $\mathrm{PhC}$ region to improve coupling efficiency [18].

The PhC waveguide was probed as shown in Fig. 2 . Alternate pulses from a $10 \mathrm{MHz}$ repetition rate fiber laser provided a train of $1550.1 \mathrm{~nm}, 14 \mathrm{ps}$ pulses at an effective rate of $5 \mathrm{MHz}$ (the maximum trigger frequency of our detection system). The pulses passed through a filtering stage consisting of a $1 \mathrm{~nm}$ bandpass filter (BPF), a circulator, and a fiber Bragg grating (FBG, $0.5 \mathrm{~nm}$, reflection $>99 \%$ ), and were TE polarized, before coupling to the chip using lensed fibers. Generated signal and idler photons at $4.8 \mathrm{~nm}$ detuning from the pump were postselected and separated using an arrayed waveguide grating (AWG), and filtered using $0.5 \mathrm{~nm}$ BPFs before being sent to InGaAs/InP single-photon detectors (SPDs, id

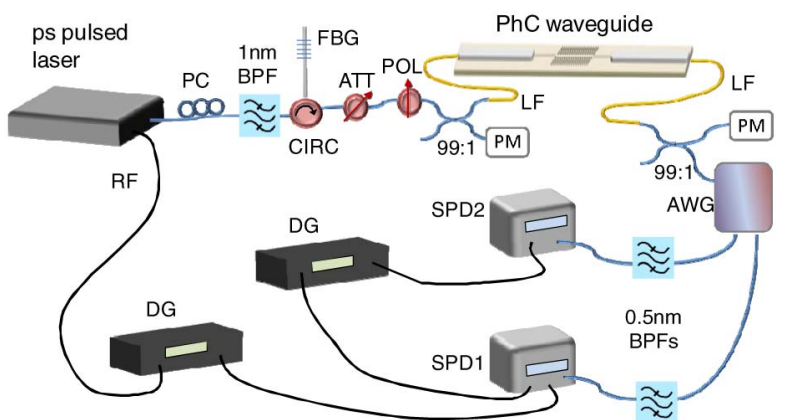

Fig. 2. (Color online) Experimental setup. RF, radio frequency signals synchronized to laser pulses; PC, polarization controller; CIRC, circulator; ATT, attenuator; POL, polarizer; LF, lensed fiber; PM, power meter; DG, delay generator.

Quantique 201). The $4.8 \mathrm{~nm}$ detuning was chosen so that the photon pair wavelengths were sufficiently far from the pump for less leaked pump noise and were within the SFWM bandwidth $(\sim 15 \mathrm{~nm})$ for efficient pair generation. The detection probability for both SPDs was set at $10 \%$ to reduce the dark counts to $4 \times 10^{-5}$ and $2 \times 10^{-5}$ per gate for idler and signal, respectively. Taking into account the waveguide output coupling loss, the AWG and BPFs insertion loss, and the detection inefficiency, the total loss of each channel was estimated to be $21.8 \mathrm{~dB}$. The simultaneous detection of a photon at both detectors was recorded as a coincidence $\left(C_{\text {raw }}\right)$. We measured accidental coincidence counts $(A)$ by changing the delay between detectors to trigger them on consecutive pulses where no photon pair correlation exists. The net coincidence count is $C=C_{\text {raw }}-A$. To improve measurement statistics, we acquired counts for $300 \mathrm{~s}$.

Figure 3 shows that the coincidences (blue diamonds) are consistently higher than the accidentals (blue circles), confirming the generation of correlated photons. It can be seen from Fig. 3 that the net coincidence (red squares) is quadratically dependent on the pump peak power $(P)$ when $P<0.42 \mathrm{~W}$, which is expected of the SFWM process. When $P \geq 0.42 \mathrm{~W}$, the net coincidence deviates from the quadratic fit (solid line) due to the onset of two-photon absorption (TPA) and the resulting free-carrier absorption (FCA) in silicon. Figure 3 also displays the measured coincidence to accidentals

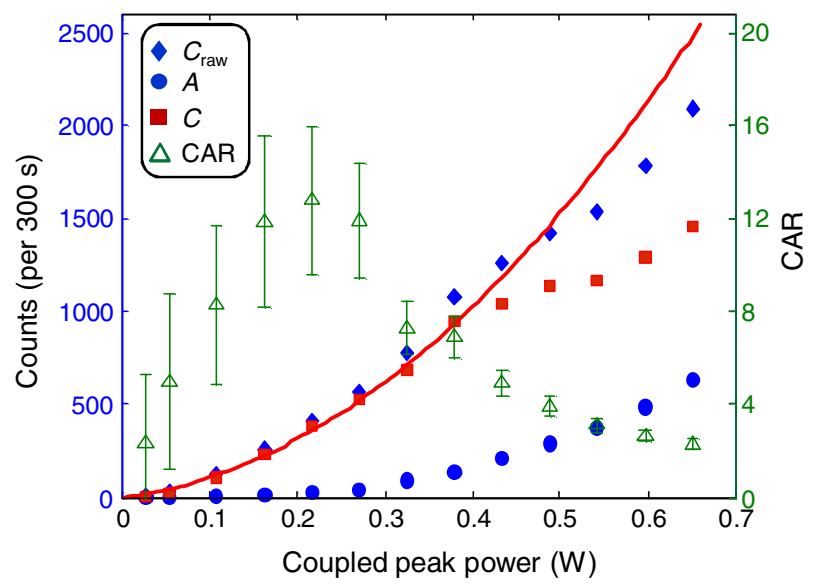

Fig. 3. (Color online) Measured coincidence $\left(C_{\text {raw }}\right)$, accidental $(A)$, net coincidence $(C)$ counts in $300 \mathrm{~s}$ and CAR as a function of coupled pump peak power. 
Table 1. Comparison of Silicon Nanowires and the PhC Waveguide

\begin{tabular}{|c|c|c|c|c|c|c|}
\hline & Estimated $\gamma[/ \mathrm{W} / \mathrm{m}]$ & Peak Power [W] & Device Length $[\mathrm{m}]$ & Measured Pairs/Pulse & Pairs $/ \mathrm{s} / \mathrm{W}^{2} / \mathrm{m}^{2} / \mathrm{nm}$ & CAR \\
\hline Ref. [12] & 100 & 0.24 & $9.11 \times 10^{-3}$ & 0.05 & $2.1 \times 10^{15}$ & 25 \\
\hline Ref. $[\overline{13}]$ & 300 & 0.05 & $1.15 \times 10^{-2}$ & 0.02 & $3.4 \times 10^{15}$ & 50 \\
\hline Our work & 4000 & 0.23 & $9.6 \times 10^{-5}$ & 0.006 & $1.8 \times 10^{18}$ & 13 \\
\hline
\end{tabular}

ratio (CAR $=C / A$, green triangles) obtained at various power levels. A maximum CAR of 12.8 is achieved for a coupled peak power of $0.23 \mathrm{~W}$. We attribute the rolloff of the CAR beyond this power level to TPA and FCA. At the pump power level for the maximum CAR, the measured net coincidence is 383 per $300 \mathrm{~s}$, corresponding to an intrinsic pair generation rate of 0.006 per pulse. Ideally, the CAR for 0.006 pairs per pulse should be $1 / 0.006=167$ if there is no noise $[11,13]$. The silicon platform does not suffer from Raman noise [13], so the CAR in our experiment was mainly limited by the pump leakage (measured to be $1.1 \times 10^{-4} /$ gate/W), afterpulsing probability $(\sim 10 \%)$, and dark counts. Improvements in future experiments will include inserting an FBG before the AWG to further suppress pump leakage, and increasing the pair photon to dark count ratio by optimizing waveguide output coupling efficiency and using low-loss filtering devices [13] Figure 3 tells us that the photon to dark count ratio cannot be enhanced by increasing pump power because of the TPA and FCA effects in silicon, which are also enhanced by slow light in the $\mathrm{PhC}$ waveguide $[16,17]$.

To compare the silicon nanowire sources and this work, we summarize the key results of Refs. $[12,13]$ in Table 1 . It can be clearly seen that the enhancement factor is over 100 in terms of pairs $/ \mathrm{s} / \mathrm{W}^{2} / \mathrm{m}^{2} / \mathrm{nm}$, having the same order of magnitude as the $\gamma^{2}$ difference.

In conclusion, we have demonstrated slow-light enhanced correlated photon pair generation in an ultracompact $96 \mu \mathrm{m}$ long silicon $\mathrm{PhC}$ waveguide. In quantum information science applications such as quantum key distribution, a source is considered to be useful when it has a CAR $>10$. Our source exceeds this specification, achieving a CAR of 12.8 at a pair generation rate of 0.006 per pulse. Operating in this low pairs per pulse regime will allow the parallel generation of many photons without contamination from multiphoton effects, where efficient heralding and routing of generated photons facilitates the creation of a pseudodeterministic single-photon source. This compact and integrated single-photon source unit offers a scalable approach to quantum information processing on-chip.

This work was supported in part by the Centre of Excellence and Federation Fellowship programs of the Australian Research Council (ARC). The silicon waveguide chip was fabricated under the Engineering and Physical Sciences Research Council (EPSRC)-UK silicon photonics consortium and supported by the European Union (EU) Seventh Framework Programme Marie Curie project "OSIRIS." A. S. Clark acknowledges a WUN Research Mobility Award and J. G. Rarity acknowledges a Royal Society Wolfson Merit Award.

\section{References}

1. N. Gisin and R. Thew, Nat. Photon. 1, 165 (2007).

2. T. D. Ladd, F. Jelezko, R. Laflamme, Y. Nakamura, C. Monroe, and J. L. O'Brien, Nature 464, 45 (2010).

3. J. Claudon, J. Bleuse, N. S. Malik, M. Bazin, P. Jaffrennou, N. Gregersen, C. Sauvan, P. Lalanne, and J.-M. Gerard, Nat. Photon. 4, 174 (2010).

4. S. Strauf, N. G. Stoltz, M. T. Rakher, L. A. Coldren, P. M. Petroff, and D. Bouwmeester, Nat. Photon. 1, 704 (2007).

5. A. L. Migdall, D. Branning, and S. Castelletto, Phys. Rev. A 66, 053805 (2002).

6. X.-S. Ma, S. Zotter, J. Kofler, T. Jennewein, and A. Zeilinger, Phys. Rev. A 83, 043814 (2011).

7. Z. Zhao, Y.-A. Chen, A.-N. Zhang, T. Yang, H. J. Briegel, and J.-W. Pan, Nature 430, 54 (2004).

8. M. Halder, A. Beveratos, N. Gisin, V. Scarani, C. Simon, and H. Zbinden, Nat. Phys. 3, 692 (2007).

9. M. Hunault, H. Takesue, O. Tadanaga, Y. Nishida, and M. Asobe, Opt. Lett. 35, 1239 (2010).

10. C. Xiong, G. D. Marshall, A. Peruzzo, M. Lobino, A. S. Clark, D.-Y. Choi, S. J. Madden, C. M. Natarajan, M. G. Tanner, R. H. Hadfield, S. N. Dorenbos, T. Zijlstra, V. Zwiller, M. G. Thompson, J. G. Rarity, M. J. Steel, B. Luther-Davies, B. J. Eggleton, and J. L. O'Brien, Appl. Phys. Lett. 98, 051101 (2011).

11. Q. Lin and G. P. Agrawal, Opt. Lett. 31, 3140 (2006).

12. J. E. Sharping, K. F. Lee, M. A. Foster, A. C. Turner, B. S. Schmidt, M. Lipson, A. L. Gaeta, and P. Kumar, Opt. Express 14, 12388 (2006).

13. K. Harada, H. Takesue, H. Fukuda, T. Tsuchizawa, T. Watanabe, K. Yamada, Y. Tokura, and S. Itabashi, IEEE J. Sel. Top. Quantum Electron. 16, 325 (2010).

14. S. Clemmen, A. Perret, S. K. Selvaraja, W. Bogaerts, D. van Thourhout, R. Baets, P. Emplit, and S. Massar, Opt. Lett. 35, 3483 (2010).

15. B. Corcoran, C. Monat, C. Grillet, D. J. Moss, B. J. Eggleton, T. P. White, L. O'Faolain, and T. F. Krauss, Nat. Photon. 3, 206 (2009).

16. C. Monat, B. Corcoran, M. Ebnali-Heidari, C. Grillet, B. J. Eggleton, T. P. White, L. O'Faolain, and T. F. Krauss, Opt. Express 17, 2944 (2009).

17. C. Monat, M. Ebnali-Heidari, C. Grillet, B. Corcoran, B. J. Eggleton, T. P. White, L. O'Faolain, J. Li, and T. F. Krauss, Opt. Express 18, 22915 (2010).

18. J. Li, L. O'Faolain, I. H. Rey, and T. F. Krauss, Opt. Express 19, 4458 (2011).

19. J. Li, T. P. White, L. O'Faolain, A. Gomez-Iglesias, and T. F. Krauss, Opt. Express 16, 6227 (2008). 